\title{
Intrapelvic trauma by metallic wire: Surgical challenges faced
}

\author{
Diogo Moura, José Mónico, João Freitas, António Figueiredo
}

\begin{abstract}
Introduction: Penetrating pelvic trauma is rare and presents as one of the most difficult challenges to trauma surgeons. Case Report: We present an unusual case of a 23-year-old male suffering a penetrating pelvic trauma by a projected metal wire. The wire was found in intrapelvic location, caused no internal organs injury and was safely removed using a Pfannenstiel extended approach. Conclusion: Preoperative assessment of foreign body position is mandatory to set-up an efficient surgical procedure. The surgeon must have a profound knowledge of pelvic anatomy in order to choose the best option for the foreign body removal without any iatrogenic lesions.
\end{abstract}

Keywords: Intrapelvic, Metallic, Removal, Trauma, Wire

\section{How to cite this article}

Moura D, Mónico J, Freitas J, Figueiredo A. Intrapelvic trauma by metallic wire: Surgical challenges faced. Case Rep Int 2015;4:52-56.

Article ID: 100018CRINTDM2015

$* * * * * * * * *$

Diogo Moura ${ }^{1}$, José Mónico ${ }^{1}$, João Freitas ${ }^{1}$, António Figueiredo $^{1}$

Affiliations: ${ }^{1}$ Centro Hospitalar e Universitário de Coimbra, Coimbra, Portugal.

Corresponding Author: Rua Fonseca Pinto, 3000-075 Coimbra, Portugal; Ph: (035) 239400 400; Email : dflmoura@ gmail.com

Received: 05 June 2015

Accepted: 31 July 2015

Published: 27 October 2015
doi:10.5348/crint-2015-18-CR-13

\section{INTRODUCTION}

Penetrating pelvic trauma is rare and presents as one of the most difficult challenges to trauma surgeons [1, 2]. Outside areas of armed conflict, penetrating injuries are responsible for fewer than $15 \%$ of traumatic deaths worldwide, but these rates vary by country [3]. There are multiple locations and courses for penetrating objects, making each case unique and demanding meticulous planning and individualized surgery [2]. Penetrating pelvic trauma patients frequently present in extreme physiological conditions, with injuries including soft tissues, pelvic bones, genitourinary system, rectum, vessels, nerves and intra-abdominal organs. The complexity of pelvic anatomy brings the potential for serious, or even lethal, damage to a spectrum of organ systems. A high index of suspicion, repeated careful evaluation and an adequate management of these injuries are mandatory to achieve a low morbidity and mortality [4].

\section{CASE REPORT}

We present a case of a 23-year-old male who was hit by a high speed metallic foreign body during the activity of lawn-mowing. The object was projected from the ground by the machine blades, entering in the medial portion of the right thigh, without any exit point. When he presented to the emergency room, he was aware and hemodynamically stable, with mild abdominal and thigh pain, but no nausea, vomiting, bowel or urinary tract symptoms or any motor or sensitive deficit of the lower extremities. The patient had pain at the superior portion of the right thigh, specifically at the entry point of the foreign body (diameter of $0.5 \mathrm{~cm}$ ).

The abdomen was moderately distended but depressible, with audible bowel sounds. There was discomfort at the deep palpation of the right lower 
quadrant, without any signs of peritoneal irritation or other symptoms. Inguinal and abdominal palpation did not reveal any abnormal presence. There were no signs of active bleeding and the rectal and urine examinations were unremarkable. Hemoglobin value was $13 \mathrm{~g} / \mathrm{dL}$ and tetanus immunization status was confirmed.

The patient brought with him an anteroposterior pelvic radiograph, performed at a primary care center, that showed a foreign body of metallic nature (Figure 1). At the emergency room, according to this radiograph, it was interpreted that the foreign body was located in the inguinal area, in a superior and anterior position in relation to the right iliopubic ramus. Taken this in consideration, a first removal attempt with an approach at the Scarpa triangle area was performed. This approach was unsuccessful, the foreign body was not even palpated. The patient was admitted to the nursery for further study of foreign body position and went through a prophylactic antibiotherapy scheme of metronidazole and ceftriaxone. The pelvic computed tomography scan (Figures 2 and 3) identified a metallic nature foreign body with 10 centimeters long situated in intrapelvic position oriented from the right obturator foramen, with a superior and posterior orientation into the right presacral musculature (S2-S3 plane). There were no signs of vascular or visceral lesions, pelvic effusion or fractures. With information regarding the exact foreign body location, collaboration of an orthopedic surgeon with experience in pelvic and acetabular trauma was requested. During the preoperative planning we asked the patient to present a metallic wire identical to the one responsible for the lesion. It was an oxidized metal wire. Before the surgical intervention, the team took fluoroscopic inlet and outlet views, so we could be certain there was no wire migration. The foreign body was in the same position as in the initial tomography images. The wire was situated at the right half of the pelvis, above the pelvic floor, so its removal access should be an endopelvic approach. We performed a suprapubic transverse laparotomy (Pfannenstiel approach) (Figure 4). After the skin and subcutaneous cellular tissue transverse incision, a vertical incision was made in the linea alba; the abdominal muscles and the urinary bladder were isolated and then, by digitoclasy, we had an extra peritoneal access to the pelvic cavity and the wire was easily visualized. The wire was removed without risks by sliding it from the original position to the entry point, following the predetermined trajectory in retrograde direction, so we could prevent iatrogenic lesions of the surrounding structures. Around the foreign body we aspirated low quantity of purulent local content. A 10 centimeter length metal wire in oxidized stage was removed (Figure 5) and the pelvic cavity was abundantly washed with normal saline and iodate solution. The tissues were sequentially closed by planes, leaving a vacuum-assisted drainage system in the previous place of the foreign body.

Postoperative course was uncomplicated and during hospital stay subcutaneous thromboprophylaxis and antibiotics were administrated. The patient was discharged after three days with prophylactic oral antibiotherapy (for the next 8 days) and analgesics.

In the follow-up consultations patient was asymptomatic, with no signs of neurologic, vascular, bowel, genitourinary lesions or others.

\section{DISCUSSION}

There are cases in literature of patients with intrapelvic foreign bodies that are asymptomatic for years [5-7]. However, to avoid serious or fatal complications (like infection and intra-abdominal lesions) caused by foreign body migration (according to Metz [8], "there are no rules for a foreign body behavior in the tissues"), its urgent but carefully planned surgical removal was indicated [9, 10]. In this case, there was high risk of infection because of the dirty ground origin and the oxidized stage of the metal wire, so the surgical removal was undoubtedly indicated. In the presence of organ lesions symptoms (such as peritonitis, hemodynamic instability, colorectal bleeding, urological lesions or others) exams like contrast abdominal radiograph, sigmoidoscopy, colonoscopy, intravenous pyelography, cystoscopy and angiography

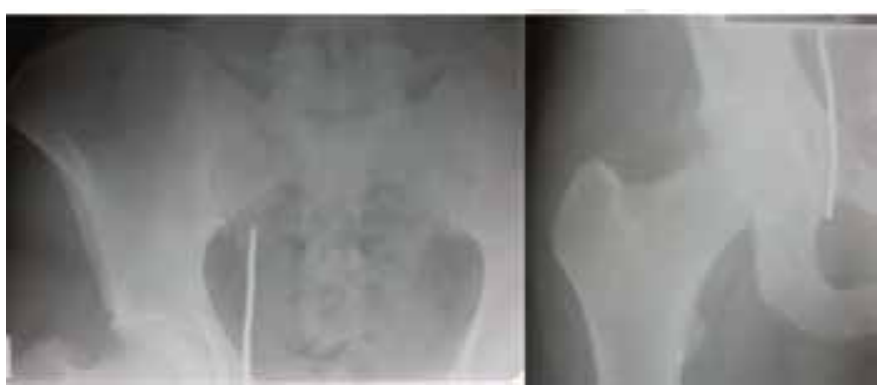

Figure 1: Pelvic radiograph demonstrating a metallic nature foreign body.

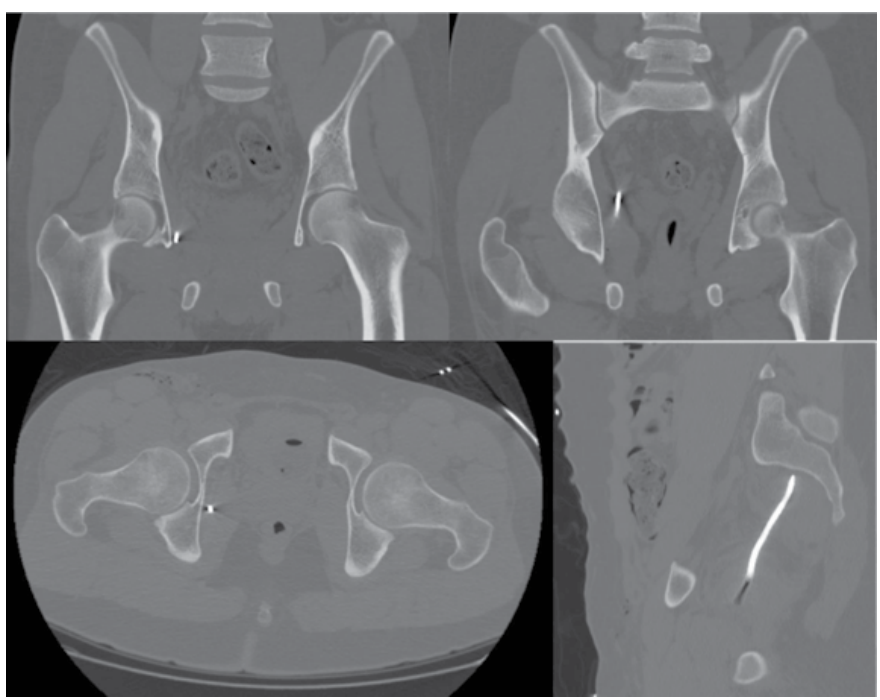

Figure 2: Pelvic computed tomography scan sagittal, coronal and lateral view showing the foreign body location. 

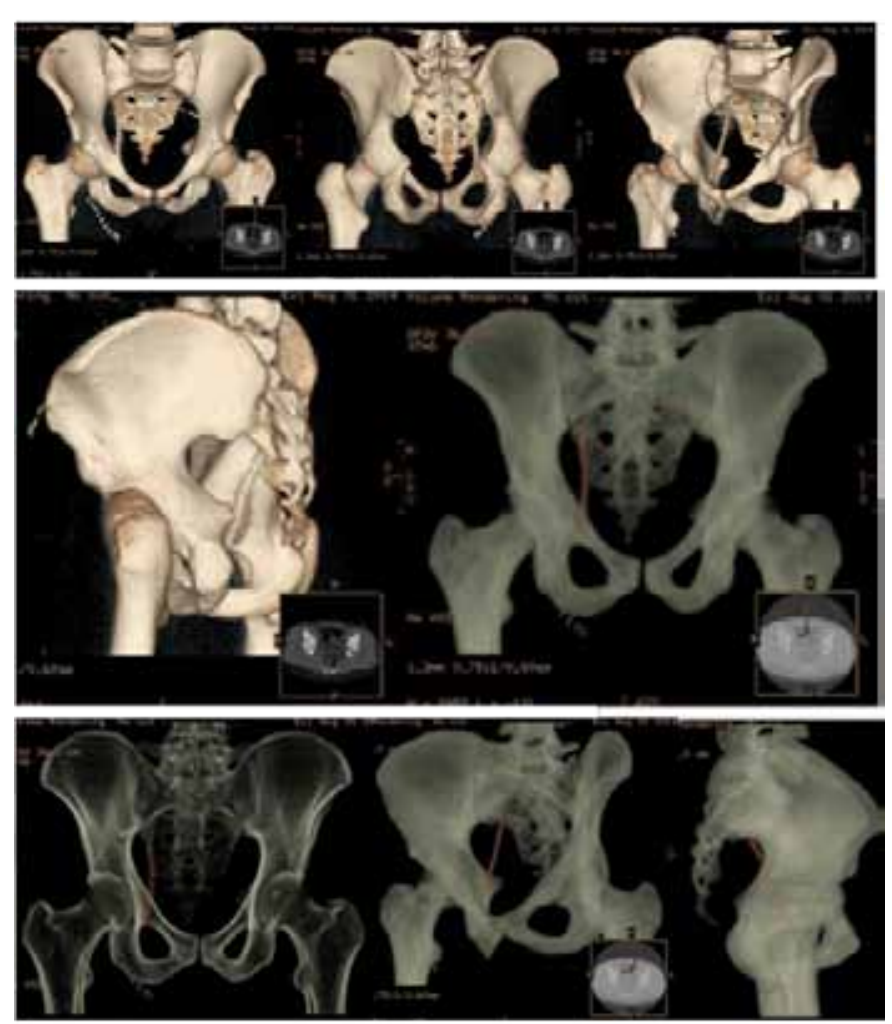

Figure 3: Three-dimensional pelvic computed tomography reconstruction. The object that appears above and for the left of the foreign body is a technique artifact.

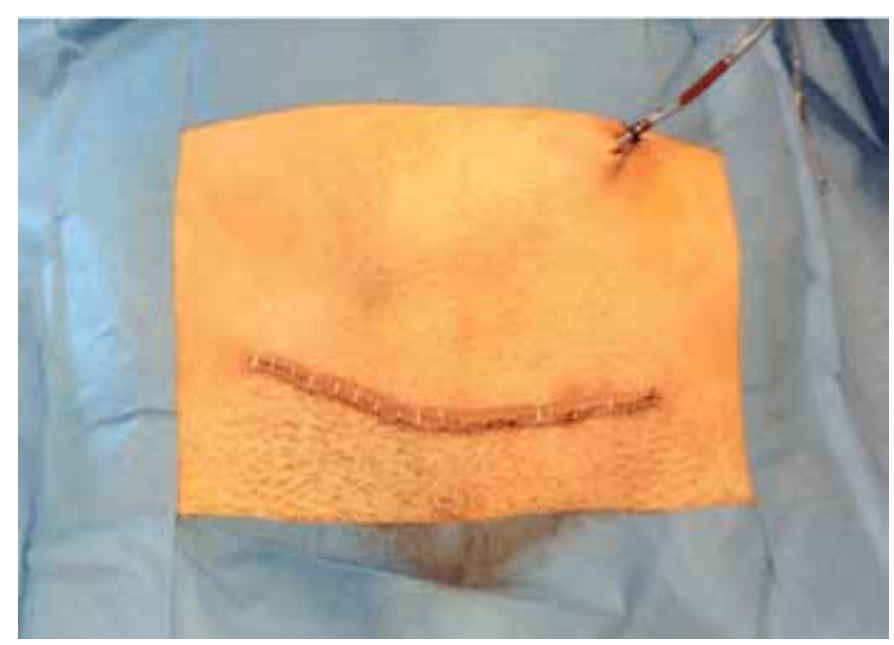

Figure 4: Pfannenstiel incision. Vacuum-assisted drainage system.

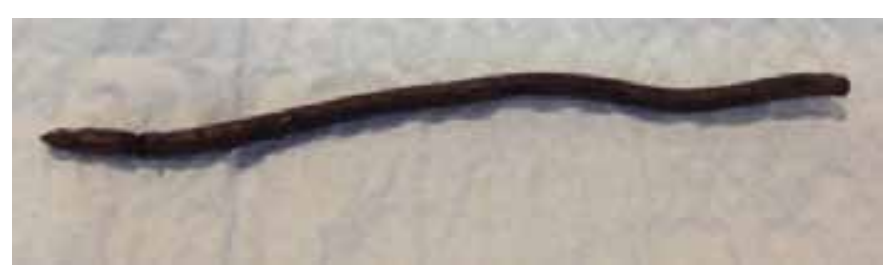

Figure 5: The removed metal wire in oxidized stage. are useful to detect, locate and characterize these lesions. When organ lesions are present urgent laparotomy and their surgical repair are mandatory [11]. In this case, we were dealing with a penetrating pelvic trauma presented by acute abdominal discomfort but without organs injury.

It is essential to perform a multiplanar detailed imaging study of the foreign body exact location because of the pelvic anatomic complexity. In order to plan the ideal surgical approach for its removal and to avoid iatrogenic lesions of the vital surrounding structures $[2,10]$. The imaging study should include anteroposterior, inlet and outlet radiograph views and a computed tomography of the pelvis. These examinations allow us to be certain of the intrapelvic foreign body position and to know its exact relation to the surrounding organs and tissues [10, 12]. An inappropriate surgical approach, particularly in complex anatomic regions like the pelvis, increases the risk of iatrogenic lesions because of insufficient visualization of the surrounding structures, making the surgical removal even more difficult $[2,10]$. Imaging findings revealed a metal wire located at the right half of the small pelvic cavity above the pelvic floor. We admitted that the projectile entered at the medial portion of the right thigh, then with superior direction went through the obturator foramen, and stopped at the small pelvic cavity. As the object was situated above the pelvic floor, an endopelvic approach was necessary. The surgeon used an approach in which he had experience in the treatment of acetabular fractures: an extended Pfannenstiel approach. This route allows an easy and secure access to the pelvic cavity by an extra peritoneal approach and the tissues are easily detachable from the pelvic walls by blunt finger dissection. Careful removal of the foreign body is advised in order to prevent lesions to the surrounding pelvic structures. Another approach option could be the wire removal beginning at Scarpa triangle (entry point) and following its trajectory with fluoroscopic control. However, this is an invasive and dangerous approach, because of the deep intrapelvic location of the wire and the neurovascular proximity during the dissection.

The penetrating pelvic trauma with metal wires is reported mostly in context of intrapelvic migration of osteosynthesis wires $[6,9,11,13,14]$, causing perforating injuries of the bowel, urinary bladder, iliac and femoral vessels. The striking features of this case report are: the rare lesion mechanism of a penetrating metal wire projected in high speed that stayed inside the pelvic cavity, the absence of intrapelvic lesions in an area with plenty of vital structures and the accurate planning and safe wire removal technique. If the foreign body is not easily accessible at the emergency room, then the patient should be referred to an expert for elective removal. Preoperative assessment of the foreign body position is always mandatory to set up an efficient surgical procedure and to avoid unsuccessful surgical approaches, like the one performed initially at the scarpa triangle. 


\section{CONCLUSION}

It is essential to make a detailed characterization of the intrapelvic foreign body position. So we can accurately plan the surgical approach and the best technique for its removal. The goal is to be as less invasive as possible and to prevent unsuccessful and risky surgical approaches. The surgeon must be an expert in the complex pelvic anatomy in order to minimize the risks and maximize the probability of a positive outcome.

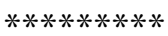

\section{Author Contributions}

Diogo Moura - Substantial contributions to conception and design, Acquisition of data, Analysis and interpretation of data, Drafting the article, Revising it critically for important intellectual content, Final approval of the version to be published

José Mónico - Analysis and interpretation of data, Revising it critically for important intellectual content, Final approval of the version to be published

João Freitas - Analysis and interpretation of data, Revising it critically for important intellectual content, Final approval of the version to be published

António Figueiredo - Analysis and interpretation of data, Revising it critically for important intellectual content, Final approval of the version to be published

\section{Guarantor}

The corresponding author is the guarantor of submission.

\section{Conflict of Interest}

Authors declare no conflict of interest.

\section{Copyright}

(C) 2015 Diogo Moura et al. This article is distributed under the terms of Creative Commons Attribution License which permits unrestricted use, distribution and reproduction in any medium provided the original author(s) and original publisher are properly credited. Please see the copyright policy on the journal website for more information.

\section{REFERENCES}

1. Arthurs $\mathrm{Z}$ et al. The use of damage-control principles for penetrating pelvic battlefield trauma. American Journal of Surgery. 2006May;191(5):604-9.

2. Hopp SJ, Culemann U, Pohlemann T. Surgical management of pelvic penetrating trauma--case report and review of literature. Injury. 2009Oct;40(10):11157.

3. Søreide K. Epidemiology of major trauma. Br J Surg. 2009 Jul;96(7):697-8.

4. Ivatury RR, Rao PM, Nallathambi M, Gaudino J, Stahl WM. Penetrating gluteal injuries. J Trauma. 1982;22:706-709.
5. Chiu WK, Hsiao CW, Kang JC, Feng JJ, Chao PC, Jao SW. Intrapelvic migration with long-term retention of a rectal thermometer: a case report. Clin Pediatr (Phila). 2007 Sep;46(7):636-8.

6. Yurtçu M, Senaran H, Türk HH, Abasiyanik A, Tuncay I. Migration of intra-articular K- wire into the contralateral pelvis after surgery for developmental dysplasia of the hip: a case report. Acta Orthop Traumatol Turc. 2010;44(5):413-5

7 Kumar S, Singh SK, Jayant K, Agrawal S, Parmar KM, Ajjoor Shankargowda S. Forgotten Kirschner wire causing severe hematuria. Case Rep Urol. 2014;2014:305868

8 Mazet R; Migration of a Kirschner wire from the shoulder region into the lung: report of two cases; J Bone Joint Surg Am, 1943Apr;25(2):477-483.

9 Kottmeier S, Born CT, Saul H. Laparoscopic retrieval of a migrating intrapelvic pin: case report and review of the literature. J Trauma. 1993Dec;35(6):952-5.

10 Zhang $P$ et al. Surgical management of penetrating pelvic trauma: a case report and literature review. Chin Journal of Traumatoly. 2012;15(6):364-6.

11 Fong YC, Lin WC, Hsu HC. Intrapelvic migration of a Kirschner wire. J Chin Med Assoc. 2005Feb;68(2):968.

12 Kircher J, Dürr H, Jansson V. Intrapelvic pin migration after periacetabular reconstruction and arthroplasty of the hip in metastatic pelvic disease: a case report. Acta Orthopaedica. 2005;76(6):941-943.

13 Posman CL, Morawa LG. Vascular injury from intrapelvic migration of a threaded pin. A case report. J Bone Joint Surg Am. 1985Jun;67(5):804-6.

14 Sayegh FE, Tsintzas D, Kapetanos GA. Intrapelvic migration of a guide pin during fixation of a hip fracture: who and what is to blame? Acta Orthop Belg.2005Apr;71(2):239-41. 
Access full text article on other devices

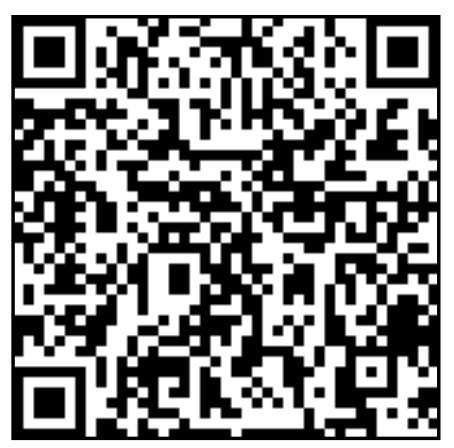

Access PDF of article on other devices

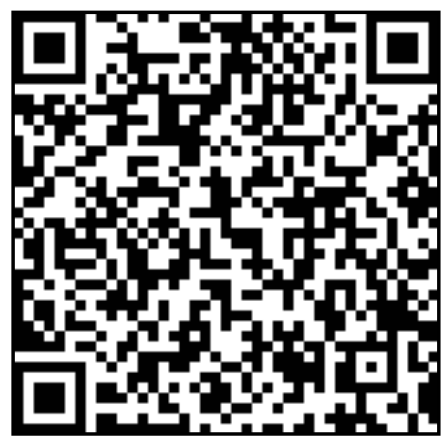

